\title{
Fièvre $Q$ chez les petits ruminants au Mali. Résultats d'une enquête sérologique
}

\author{
S.S. Sidibe ${ }^{1 *}$ K.W. Coulibaly ${ }^{1 *}$ M. Dakouo $^{1}$ \\ Z. Tarnagda ${ }^{2}$ A. Sery ${ }^{1}$ M. Niang ${ }^{1}$ K. Traore ${ }^{1}$ \\ H. Nantoume ${ }^{3}$ S. Diarra ${ }^{4}$ H. Seyni ${ }^{5}$
}

Mots-clés

Ovin - Caprin - Fièvre Q Reproduction - Perte - Sérologie Mali.

\begin{abstract}
Résumé
Au Mali, les pertes en reproduction constituent des contraintes majeures à I'amélioration de la productivité de l'élevage des petits ruminants. Parmi les causes de ces pertes qui demeurent mal connues figurent, entre autres, l'infertilité, les avortements, l'orchite et la chétivité. L'objet de la présente étude a été d'évaluer la prévalence sérologique de la fièvre $Q$ dans les élevages de petits ruminants où des cas de pertes en reproduction ont été observés, ainsi que la valeur financière des pertes liées aux avortements enregistrés. L'étude a été conduite entre 2006 et 2009 dans les zones agropastorales des localités de Bougouni (région de Sikasso), Nioro (région de Kayes), Kéniébougouwéré (région de Ségou) et Koro (région de Mopti). Sur les 718 sérums analysés par la méthode Elisa indirecte, 155 (21,5 \pm 3 p. 100) se sont révélés positifs aux anticorps de Coxiella burnetii. Cette prévalence a varié en fonction des sites et du rang de portée. La prévalence la plus élevée a été observée à Kéniébougouwéré (35 \pm 6 p. 100), suivi de Nioro ( $28,5 \pm 7,5$ p. 100), puis de Bougouni $(10,8 \pm 4,6$ p. 100), et la plus faible à Koro $(5,8 \pm 3,7$ p. 100). Des études complémentaires intégrant le diagnostic moléculaire (technique PCR) pourraient aider à élucider le rôle étiologique de cette pathologie dans les cas de pertes en reproduction couramment enregistrés chez les petits ruminants au Mali. L'analyse technico-économique a permis d'évaluer la valeur financière des pertes.
\end{abstract}

\section{INTRODUCTION}

La fièvre $Q$ est une zoonose qui entraine des pertes en reproduction (1) dans les élevages de petits ruminants avec des conséquences économiques non négligeables. En effet, cette maladie a été mise en évidence dans tous les pays du monde où elle a été

\footnotetext{
1. Laboratoire central vétérinaire, BP 2295, Bamako, Mali.

2. Institut de recherche en sciences de la santé, Bobo Dioulasso, Burkina Faso.

3. Centre régional de recherche agronomique de Sotuba, Bamako, Mali.

4. Annexe de l'Institut polytechnique rural / Institut de formation et de recherche appliquée de Katibougou, Bamako, Mali.

5. Centre international de recherche-développement sur l'élevage en zone subhumide, Bobo-Dioulasso, Burkina Faso.

* Auteurs pour la correspondance

Tél./fax : +2232243344

Portable : +223760527 85; e-mail : sidibsatigui@yahoo.fr

Portable : +22375 1976 85; e-mail :welecoulibaly@yahoo.fr
}

recherchée $(22,31)$, à l'exception de la Nouvelle-Zélande $(13,40)$ Elle est mieux connue dans les pays développés et dans certains pays en développement. Des études réalisées notamment en Mauritanie en 1988 (7), en Tunisie en 2005 dans les troupeaux sédentaire et nomade de petits ruminants (28), au Tchad chez les petits ruminants en 2004 (37) ont confirmé sa présence chez les petits ruminants.

La fièvre $\mathrm{Q}$ est causée par la bactérie ubiquitaire Coxiella burnetii et affecte surtout les caprins et les ovins $(10,26)$. Des avortements peuvent survenir chez les femelles au cours du dernier mois de gravidité (21), en majorité chez les primipares (34). La naissance de chevreaux et d'agneaux chétifs mourant quelques jours après la naissance est aussi fréquente (34) avec parfois des rétentions placentaires (21). Chez les ovins, la maladie peut se manifester aussi par des pneumonies avec ou sans signes oculaires de kérato-conjonctivite (15). Les ruminants domestiques constituent le principal réservoir de la maladie $(12,27,31)$. L'excrétion de Coxiella se fait par 
les fèces, l'urine, les sécrétions nasales, le lait, ainsi que le placenta et les sécrétions vaginales lors de la mise bas et d'avortements (33). La transmission de la maladie se fait par voie respiratoire (par inhalation d'aérosols ou de poussières souillées) et également dans une moindre mesure par voie buccale. Il existe une possibilité de transmission vectorielle par des tiques (16). Chez certaines tiques, une transmission transovarienne (18) et trans-stadiale (9) a été observée. L'homme se contamine surtout suite à l'inhalation de poussières infectées $(17,21,30,38)$ par contact avec les produits contaminés et probablement par consommation de lait non pasteurisé ou de produits laitiers crus $(5,30)$. Les caprins et les ovins constituent la principale source de contamination (2). L'homme peut aussi se contaminer à travers des contacts avec des chiennes et des chattes domestiques $(6,19,24)$ en période de peripartum. Le risque d'une contamination à partir d'animaux sauvages n'est pas totalement exclu, notamment parce que les rongeurs (12), les oiseaux (38) et les lagomorphes (20) peuvent être porteurs de la bactérie.

Le diagnostic de laboratoire de la fièvre $Q$ repose habituellement sur la sérologie et plus récemment sur la technique d'amplification en chaîne par polymérase (PCR) plutôt que sur la recherche du germe infectant lui-même. La culture de $C$. burnetii est techniquement difficile et n'est pas fréquemment réalisée en diagnostic de routine dans la plupart des laboratoires de microbiologie. La détection moléculaire de l'ADN bactérien est recommandée de plus en plus par les laboratoires spécialisés. Le diagnostic doit s'appuyer sur l'analyse sérologique d'une dizaine de sérums de femelles ayant avorté ou mis bas récemment en association avec une recherche directe de l'agent étiologique (16). Lorsque ce diagnostic est positif, tout le troupeau est considéré comme infecté. Selon les antigènes, différents anticorps contre les bactéries de phases I et II sont détectés. A cet effet, plusieurs techniques sérologiques permettent de détecter la fièvre $\mathrm{Q}$ : la technique de référence est celle de l'immunofluorescence indirecte (22) avec titrage des immunoglobulines ( $\operatorname{IgG}, \operatorname{IgM}, \operatorname{IgA})$ dirigées contre un antigène en phase I et II (9). Mais elle n'est pas simple d'utilisation. La technique de fixation du complément est la plus utilisée en médecine vétérinaire, notamment chez les petits ruminants. Cette technique ne détecte que les anticorps en phase I. Elle est peu spécifique et peut donner des résultats négatifs même chez des animaux excrétant massivement $C$. burnetii (9). La méthode de choix est l'Elisa qui est à la fois plus sensible et plus spécifique que la méthode de fixation du complément et plus simple que l'immunofluorescence. Compte tenu de la variation de la cinétique des anticorps chez les chèvres $(21)$ et chez les moutons $(10,17,26)$ et d'une réaction différente face à la souche de l'antigène pour le test, il est préférable d'utiliser la technique Elisa chez les petits ruminants (29).

Au Mali, l'élevage des petits ruminants constitue une des principales activités génératrices de revenus pour les populations rurales. Cependant, au nombre des contraintes à l'amélioration de la productivité des petits ruminants figurent entre autres les nombreux cas de pertes en reproduction (avortements, mortinatalité, infertilité, orchite, chétivité) dont sont responsables certaines maladies abortives comme la fièvre Q (8). Cette pathologie a un caractère zoonotique et demeure négligée (absence de dépistage systématique et de stratégie adaptée de lutte). L'évaluation de l'importance hygiénique de la maladie n'a pas été abordée dans cet article et fera l'objet d'une autre étude.

Les données disponibles sur ce sujet au Mali et dans la sous-région sont parcellaires. La prévalence de cette pathologie abortive y demeure mal connue chez les petits ruminants ; par ailleurs, son importance économique n'a jamais été étudiée chez ces animaux.

Une étude réalisée en 1999 sur des ovins dans le district de Bamako a permis d'établir que l'agent étiologique de la fièvre Q circule chez cette espèce dans le pays (3). En 2003, une autre étude effectuée chez des humains fébriles à Bamako et à Mopti en vue d'évaluer l'importance de la brucellose et de la fièvre Q liées à la consommation de lait et de produits laitiers a donné un taux d'infection global de 50 p. 100 pour la fièvre Q (36 p. 100 à Bamako et 61,9 p. 100 à Mopti) (4).

$\mathrm{Au}$ regard des particularités épidémiologiques inhérentes à la fièvre $Q$ et de la faiblesse des premières investigations menées au Mali, la présente étude a eu pour but d'évaluer la prévalence sérologique de la fièvre $Q$ chez les petits ruminants, de vérifier l'existence de liens avec les principaux facteurs de risque (site, espèce de petits ruminants, âge, rang de portée, nature de la perte en reproduction et saison de l'année), et d'évaluer la valeur financière des pertes en reproduction liées à cette pathologie (avortements).

\section{MATERIEL ET METHODES}

\section{Sites de l'étude et conduite d'élevage}

Les quatre sites de l'étude étaient localisés dans les régions de Kayes, Sikasso, Ségou et Mopti. La représentativité numérique et génétique du cheptel de petits ruminants a justifié le choix de ces régions : la population de petits ruminants représente 45 p. 100 de l'effectif national du Mali et toutes les races de petits ruminants élevées dans le pays y sont présentes. Avant de sélectionner les sites de l'étude, une mission de prise de contact et d'échange avec les structures d'encadrement et les communautés d'agroéleveurs a permis de retenir et de visiter les cercles, arrondissements et villages qui hébergeaient les effectifs les plus importants de petits ruminants, dans lesquels prédominait l'élevage sédentaire. Ainsi, le choix définitif des sites a été fixé en collaboration avec les structures techniques régionales et locales d'encadrement en fonction des informations disponibles sur l'importance des effectifs de petits ruminants, l'incidence des cas de pertes en reproduction, l'accessibilité du site en toute saison et le degré de réceptivité des éleveurs aux innovations technologiques (figure 1).

Situé dans le cercle (division administrative) de Ségou, le site de Kéniébougouwéré a un climat sahélien avec une pluviométrie variant entre 400 et $800 \mathrm{~mm}$. La végétation est composée d'arbustes et d'épineux, et la savane est arborée.

Le site de Bougouni est situé en zone soudanienne et la pluviométrie y varie entre 800 et $1110 \mathrm{~mm}$ par an. La végétation est de type savane et le vent dominant est l'harmattan. Dans cette zone, pendant la saison sèche, les animaux sont généralement en divagation et pâturent aux alentours du village pendant la journée et rejoignent la concession le soir. En revanche, pendant l'hivernage, ils sont en stabulation afin de protéger les cultures (enclos, cases). Les animaux des troupeaux de petite taille sont attachés aux piquets pendant la journée dans les pâturages disponibles et conduits le soir dans les enclos ou cases localisés dans les familles.

Nioro se trouve en zone sahélienne où la pluviométrie varie entre 500 et $700 \mathrm{mn}$ par an. Les vents dominants sont l'harmattan et la mousson. La végétation est composée essentiellement d'acacias et d'espèces épineuses.

Koro est situé en zone sahélienne chaude et sèche où la pluviométrie varie entre 350 et $500 \mathrm{~mm}$ par an. La végétation est composée de baobabs, de caïlcédrats et d'espèces épineuses. Dans la zone sahélienne, les agroéleveurs ne construisent pas d'enclos ni de cases pour les animaux dans la majorité des cas. Ces derniers sont souvent attachés aux piquets dans la cour de la concession ou, le plus souvent, ils sont conduits dans des troupeaux collectifs par les bergers. 


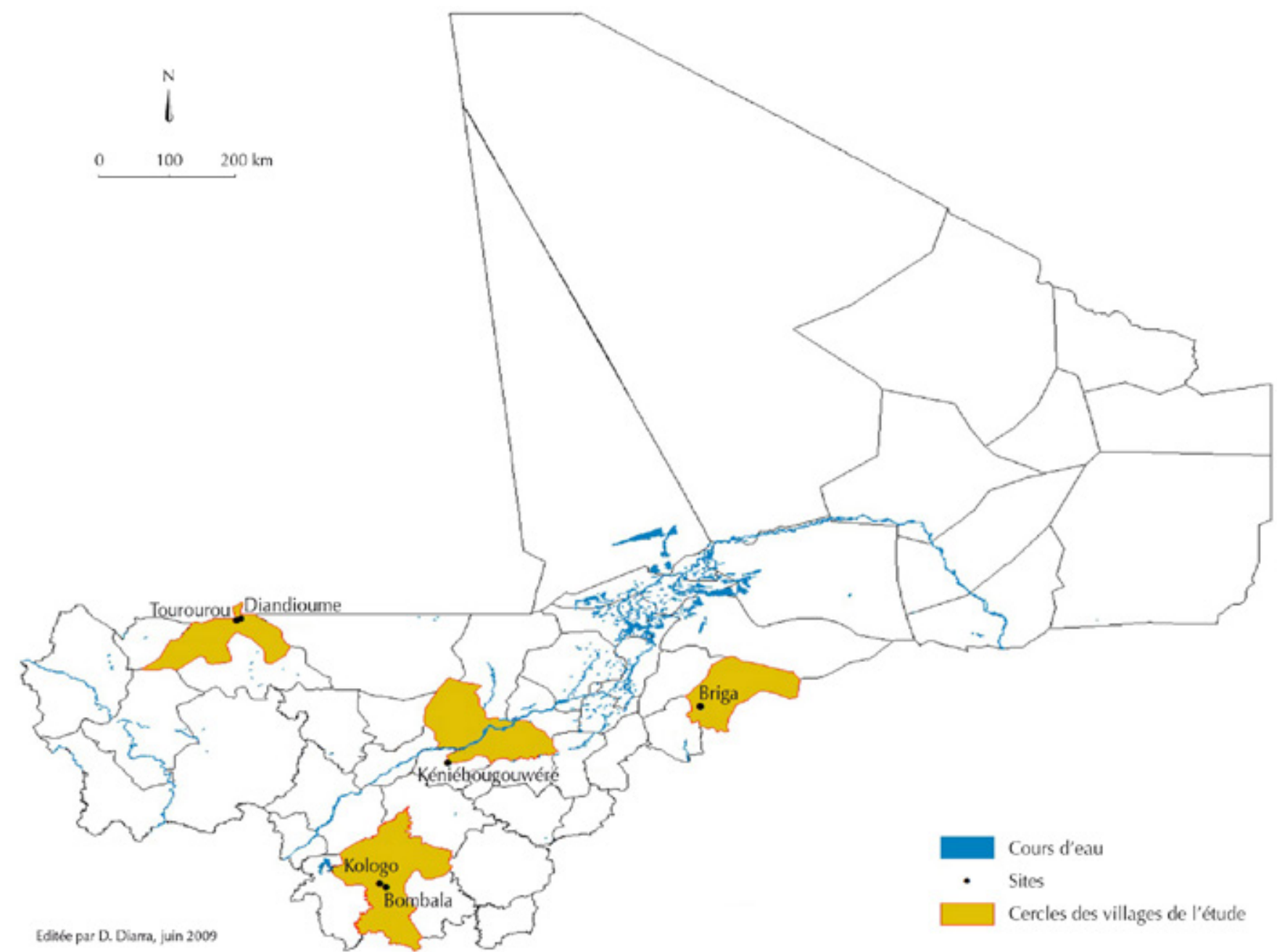

Figure 1 : situation géographique des différents sites de l'étude.

\section{Animaux}

Le cheptel des petits ruminants des quatre sites a été estimé à 7592 animaux répartis comme suit : 2709 à Kéniébougouwéré, 3166 à Nioro, 872 à Bougouni et 845 à Koro. L'ensemble du cheptel mentionné a fait l'objet de suivi pendant la durée de l'étude. Les brebis étaient de races Djallonké, Maure, Peulhe et Bali-Bali, et des métisses issues de croisements entre les diverses races ovines. Les caprins étaient des chèvres du Sahel, des chèvres Naines (Djallonké) et des métisses issus de différents croisements.

Les animaux avaient entre deux mois et huit ans et étaient répartis comme suit : 98 (13,6 p. 100) avaient de 2 mois à 1 an et étaient non reproducteurs ; $552(76,9$ p. 100) avaient de 1 à 4 ans et étaient en âge de reproduction ; 68 (9,5 p. 100) avaient 4 ans et plus et étaient très certainement reproducteurs.

\section{Saisonnalité}

Afin d'étudier la saisonnalité de la maladie, les animaux ont été suivis pendant deux ans (de novembre 2007 à octobre 2009), couvrant ainsi les différentes saisons de l'année : saison sèche froide (de novembre à février), saison sèche chaude (de mars à juin) et saison pluvieuse (de juillet à octobre).

\section{Suivi et prélèvements}

Un suivi quotidien des animaux et des troupeaux a été réalisé par des observateurs (agents d'élevages privés). Un observateur a été recruté par site d'étude et initié aux techniques de collecte de prélèvements et de conditionnement pour leur expédition au laboratoire. La supervision des activités des observateurs a été assurée par l'équipe de recherche avec une fréquence trimestrielle.

Les prélèvements sanguins ont été réalisés uniquement sur les animaux ayant présenté une des pertes en reproduction. Au total 718 petits ruminants ont été prélevés, dont 422 caprins $(58,8$ p. 100) et 296 ovins $(41,2$ p. 100). Pour chaque prélèvement, les informations relatives à l'espèce, soit la race, l'âge et le rang de portée pour les femelles, ont été notées. Les animaux identifiés ont été examinés (thermométrie, recherche d'autres signes cliniques comme le jetage, la toux, la salivation, les diarrhées), puis prélevés à la veine jugulaire à l'aide d'un tube Vacutainer sous vide et stérile. Les sérums ont été récoltés le même jour, conservés au frais jusqu'à leur expédition sous glace au Laboratoire central vétérinaire pour les analyses. Compte tenu de la prédominance des femelles, il n'a pas été opportun d'étudier le lien pouvant exister entre le taux d'infection et le sexe de l'animal. Les données clinico-épidémiologiques ont été enregistrées à travers le recensement systématique de tous les cas de pertes en reproduction (avortement, mortinatalité, infertilité, orchite et chétivité).

\section{Diagnostic de laboratoire}

La technique PCR n'étant pas disponible en routine pour son coût élevé pour les laboratoires du pays, la technique Elisa a été privilégiée par rapport à d'autres techniques de diagnostic sérologiques 
(35). Plusieurs auteurs affirment qu'elle est recommandée dans le dépistage sérologique de la fièvre $\mathrm{Q}$ chez l'espèce ovine et caprine compte tenu de sa rapidité et de sa sensibilité et spécificité élevées $(11,29,35)$.

Tous les sérums de la présente étude ont été analysés à l'aide d'un kit Elisa (Idvet, Montpellier, France). La lecture des plaques a été faite à $450 \mathrm{~nm}$ à l'aide d'un lecteur de plaques (Titreteck Multiscan Plus). Ceci a permis de détecter les infections récentes et anciennes par la mise en évidence des IgM et IgG. Les résultats des analyses ont été interprétés selon les recommandations du fabricant. Les cas douteux ont été testés à nouveau afin d'être mieux fixé sur leur statut sérologique.

\section{Analyse de l'importance économique de la fièvre $Q$}

Afin d'évaluer l'impact économique de la maladie, des données techniques et économiques ont été collectées dans les différents sites à travers l'administration de questionnaires par interviews selon le modèle de Hanon et coll. (36). Ces données ont concerné les effectifs totaux de petits ruminants, les effectifs moyens par éleveur, le poids et le prix moyens des petits ruminants, les quantités de lait produites, la durée de lactation, les prix moyens du litre de lait, les quantités de lait et le nombre d'animaux perdus à la suite d'avortements.

Les pertes économiques engendrées par la perte de lait causée par les avortements ont été évaluées en multipliant les volumes totaux de lait perdu par le prix moyen du litre au niveau du site.

Les pertes économiques engendrées par la perte d'animaux causée par les avortements ont été évaluées en multipliant le nombre total d'avortements par le prix moyen des petits ruminants au sein du site, d'après la formule :

prix unitaire de l'animal $=$ (prix moyen mâle + prix moyen femelle) / 2

L'impact économique global a été évalué en additionnant les valeurs monétaires (en francs CFA) de toutes les pertes (lait, avortement) :

pertes totales $=$ pertes en lait + pertes en petits ruminants (avortements)

\section{Analyse statistique}

L'analyse statistique a été faite avec le logiciel Stata version 6. La régression logistique a été utilisée pour l'analyse des données. Les différences ont été considérées comme significatives lorsque $\mathrm{p} \leq 0,05$. Les facteurs pris en compte pour vérifier le lien avec la maladie ont été le site d'étude, l'espèce de petit ruminant, l'âge, la nature de la perte en reproduction, le rang de portée et la saison.

\section{RESULTATS}

L'analyse des 718 sérums par la technique Elisa indirecte a révélé 155 sérums positifs pour la fièvre Q, soit une séroprévalence de $21,5 \pm 3$ p. 100 (tableau I). Les analyses statistiques ont montré qu'il existait un lien significatif entre les facteurs site et rang de portée, et la maladie ( $<<0,001)$. Cependant, aucun lien n'a été établi entre les autres facteurs (espèce, classe d'âge, nature de la perte, saison) et la maladie.

La séroprévalence de la maladie a varié en fonction du site d'étude et a été la plus élevée à Kéniébougouwéré $(35 \pm 6$ p. 100) (tableau I).
Le tableau II montre la variabilité de la séroprévalence de la fièvre Q selon le rang de portée.

Les taux d'infection les plus élevés ont été observés chez les animaux de troisième portée, suivi de ceux des sixième et cinquième portées. Les animaux appartenant aux rangs de portées 0,7 et 8 n'ont pas été pris en compte en raison de la faiblesse du nombre de cas de pertes en reproduction enregistré. Le rang de portée a été statistiquement associé à la maladie ( $<<0,001)$ (tableau III).

\section{Tableau}

Séroprévalence de la fièvre Q en fonction du site

\begin{tabular}{lrrrrr} 
Site & \multicolumn{2}{c}{ Sérum } & & $\begin{array}{c}\text { Prévalence } \\
\mathbf{( \% )}\end{array}$ & IC * \\
\cline { 2 - 5 } & Testé & Négatif & Positif & & \\
\hline Kéniébougouwéré & 248 & 161 & 87 & 35,0 & $29,1-41,0$ \\
Nioro & 140 & 100 & 40 & 28,5 & $21,0-36,0$ \\
Bougouni & 175 & 156 & 19 & 10,8 & $6,2-15,4$ \\
Koro & 155 & 146 & 9 & 5,8 & $2,1-9,5$ \\
\hline Total & 718 & 563 & 155 & 21,5 & $18,5-24,6$
\end{tabular}

* Intervalle de confiance à $95 \%$

\section{Tableau II}

Prévalence de la fièvre $Q$ en fonction de la portée

\begin{tabular}{lrrrrr} 
Rang de portée & \multicolumn{3}{c}{ Sérum } & \multicolumn{3}{c}{$\begin{array}{c}\text { Prévalence } \\
(\%)\end{array}$} & IC * \\
\cline { 2 - 5 } & Testé & Négatif & Positif & & \\
\hline $1^{\mathrm{er}}$ & 85 & 69 & 16 & 18,8 & $10,5-27,1$ \\
$2^{\mathrm{e}}$ & 59 & 47 & 12 & 20,3 & $10,0-30,6$ \\
$3^{\mathrm{e}}$ & 38 & 26 & 12 & 31,5 & $16,8-46,3$ \\
$4^{\mathrm{e}}$ & 31 & 27 & 4 & 12,9 & $1,1-24,7$ \\
$5^{\mathrm{e}}$ & 13 & 10 & 3 & 23,0 & $0,1-45,9$ \\
$6^{\mathrm{e}}$ & 14 & 10 & 4 & 28,5 & $4,9-52,2$ \\
\hline & 251 & 199 & 52 & 20,7 & $15,7-25,7$
\end{tabular}

* Intervalle de confiance à $95 \%$

\section{Tableau III}

Résultats d'analyse statistique (rang de portée)

\begin{tabular}{lccc} 
Variable & Coefficient & $\mathbf{P}$ & IC $*$ \\
\hline Portée 1 & $-2,673$ & $<0,001$ & $-3,602--1,744$ \\
Portée 2 & $-4,122$ & $<0,001$ & $-5,607--2,637$ \\
Portée 3 & $-3,968$ & $<0,001$ & $-5,461--2,475$ \\
Portée 4 & $-3,734$ & $<0,001$ & $-5,241--2,228$ \\
Portée 5 & $-2,464$ & $<0,001$ & $-3,845--1,083$ \\
Portée 6 & $-3,275$ & $<0,001$ & $-5,426--1,123$ \\
Constance & 1,483 & $<0,001$ & $1,099-1,867$
\end{tabular}

* Intervalle de confiance à $95 \%$ 
L'analyse sérologique selon l'espèce de petit ruminant a montré la prévalence la plus élevée chez les caprins avec 105 cas positifs sur 422 sérums testés $(24,9 \pm 4,1$ p. 100), contre 50 cas positifs sur 296 sérums testés $(16,9 \pm 4,3$ p. 100) chez les ovins.

La prévalence la plus élevée a été enregistrée chez les animaux âgés de plus de quatre ans avec 20 cas positifs sur 68 testés $(29 \pm 11$ p. 100). Chez les animaux âgés de 1 à 4 ans, 118 cas positifs ont été enregistrés sur 552 testés $(21,4 \pm 3,4$ p. 100). En outre, la prévalence a été beaucoup moins importante chez les animaux âgés de moins d'un an avec 17 cas positifs sur 98 testés $(17,3 \pm 7,5$ p. 100).

La prévalence la plus élevée a été enregistrée pendant la saison pluvieuse avec 64 cas positifs sur 276 analysés $(23,1 \pm 4,9$ p. 100). Pendant la saison sèche froide, 51 animaux se sont révélés positifs sur 251 testés $(20,3 \pm 4,9$ p. 100$)$. Pendant la saison sèche chaude, 40 animaux ont été trouvés positifs sur 191 testés $(20,9 \pm 5,7$ p. 100).

Sur 590 cas d'avortement observés, des anticorps anti-fièvre Q ont été mis en évidence dans 140 cas $(23,7 \pm 3,4$ p. 100). Parmi les 78 animaux chétifs, 10 ont été positifs pour la maladie $(12,8 \pm 7,2$ p. 100), et trois animaux infertiles sur 36 ont été positifs $(8,4 \pm 9,0$ p. 100). Parmi les huit animaux chez lesquels des cas de rétention placentaire ont été observés, deux ont été positifs ( $25 \pm 30,1$ p. 100). En revanche, les six animaux portant des signes d'orchite ont été négatifs.

Les résultats de l'évaluation de l'impact économique de la fièvre Q sont résumés dans le tableau IV. Le nombre d'élevages suivis et le nombre moyen d'animaux par élevage a différé en fonction du site. La production moyenne journalière de lait par animal a été de 0,32 litre. Elle a été plus importante à Kéniébougouwéré avec 0,36 litre et plus faible à Nioro avec 0,25 litre. Le litre de lait de petit ruminant était vendu plus cher à Nioro (400 FCFA/litre) qu'à Bougouni (115 FCFA). Dans tous les sites, le lait produit était surtout destiné à l'autoconsommation, expliquant l'absence de données sur la vente de lait et les quantités consommées (tableau IV). Les races dominantes des moutons et des chèvres selon les sites ont été les suivantes : Djallonké à Bougouni, Djallonké et Bali-Bali à Koro, Sahel à Ségou et à Nioro.

L'estimation par site des pertes économiques a été faite pour le lait perdu et pour la perte d'animaux (avortements) (tableau V). Au

\section{Tableau IV}

Evaluation par site de l'impact économique de la fièvre Q

\begin{tabular}{|c|c|c|c|c|c|c|}
\hline \multirow{2}{*}{\multicolumn{2}{|c|}{ Paramètre technico-économique }} & & \multicolumn{4}{|c|}{ Site de l'étude } \\
\hline & & \multicolumn{2}{|r|}{ Bougouni } & Koro & Kéniébougouwéré & Nioro \\
\hline \multicolumn{3}{|c|}{ Effectif de petits ruminants (PR) } & 872 & 845 & 2709 & 3166 \\
\hline \multicolumn{3}{|l|}{ Nb. d'élevages } & 44 & 88 & 67 & 76 \\
\hline \multicolumn{3}{|c|}{ Nb. moyen de PR/éleveur } & 20 & 46 & 40 & 42 \\
\hline \multicolumn{3}{|c|}{ Poids moyen ovin mâle (kg) } & 35 & 37 & 35 & 43 \\
\hline \multicolumn{3}{|c|}{ Poids moyen ovin femelle (kg) } & 27 & 29 & 27 & 32 \\
\hline \multicolumn{3}{|c|}{ Prix moyen mâle (FCFA) } & 24000 & 36000 & 30850 & 26850 \\
\hline \multicolumn{3}{|c|}{ Prix moyen femelle (FCFA) } & 15000 & 21000 & 20000 & 18500 \\
\hline \multicolumn{3}{|c|}{ Production de lait/jour (L) } & 0,35 & 0,32 & 0,36 & 0,25 \\
\hline \multicolumn{3}{|c|}{ Durée production lait (jour) } & 60 & 60 & 60 & 60 \\
\hline \multicolumn{3}{|c|}{ Prix moyen / litre lait (FCFA) } & 115 & 225 & 200 & 400 \\
\hline Vente de lait & & & $N D^{*}$ & ND & ND & ND \\
\hline Consommation & & & ND & ND & ND & ND \\
\hline \multicolumn{4}{|c|}{ Production moyenne journalière de lait/animal (L) } & \multicolumn{2}{|l|}{0,32} & \\
\hline \multicolumn{7}{|l|}{ * Non déterminé } \\
\hline \multirow{2}{*}{\multicolumn{7}{|c|}{ Estimation des pertes économiques en animaux et en lait par site suite aux avortements }} \\
\hline & & & & & & \\
\hline Sites & $\begin{array}{l}\text { Nb. animaux } \\
\text { perdus }\end{array}$ & $\begin{array}{l}\text { Prix moyen / } \\
\text { animal }\end{array}$ & $\begin{array}{c}\text { Valeur pertes } \\
\text { d'animaux (FCFA) }\end{array}$ & $\begin{array}{l}\text { Quantité lait } \\
\text { perdue / (L) }\end{array}$ & $\begin{array}{l}\text { Prix / litre lait } \\
\text { (FCFA) }\end{array}$ & $\begin{array}{l}\text { Valeur pertes } \\
\text { en lait (FCFA) }\end{array}$ \\
\hline Kéniébougouwéré & 84 & 50850 & 4271400 & 2117 & 200 & 423400 \\
\hline Nioro & 42 & 45350 & 1904700 & 905,75 & 400 & 362300 \\
\hline Koro & 8 & 28500 & 228000 & 562 & 115 & 64630 \\
\hline Bougouni & 26 & 19500 & 507000 & 562 & 225 & 126450 \\
\hline Sous-totaux & 160 & & 6911100 & 4146,7 & & 976780 \\
\hline Valeur totale & & & & 788788 & & \\
\hline
\end{tabular}


total 160 animaux ont été perdus suite aux avortements. Le coût de ces pertes s'est élevé à 6911100 FCFA. La valeur monétaire des pertes en lait dans la même zone s'est élevée à 976780 FCFA. Le coût total des pertes ainsi enregistrées pendant la période de l'étude a été estimé à 7888880 FCFA.

\section{- DISCUSSION}

Avec la technique Elisa, certaines brebis excrétrices de coxielles par voie vaginale seraient négatives alors que d'autres seraient positives mais n'excrèteraient pas la bactérie et ne subiraient pas d'avortement (30). Cette technique permet de détecter aussi bien les infections anciennes que les nouvelles, et de nombreuses séroconversions dans un effectif indiquent la circulation de la bactérie dans le cheptel, sans qu'il y ait forcément de symptômes évocateurs de la maladie. Elle ne permet pas d'estimer l'importance de l'excrétion bactérienne. Un animal séropositif n'est pas forcément excréteur, un autre peut être séronégatif et excréteur (14). Ainsi, l'examen sérologique est d'une interprétation délicate dans le cas de la fièvre Q. La technique Elisa aurait pu être associée à une méthode de recherche directe de l'agent étiologique comme la PCR qui est une technique très sensible (16), même si avec cette dernière des résultats positifs sont possibles alors que la bactérie n'est plus active. Pour être complète, l'étude devrait associer le diagnostic clinique et microbiologique direct au diagnostic sérologique.

Le taux de séroprévalence global de 21,5 p. 100 a été inférieur à celui obtenu par d'autres auteurs lors d'une étude prospective (3) chez les ovins dans le district de Bamako (32 p. 100). Ce phénomène pourrait s'expliquer par divers facteurs, notamment la taille plus grande de l'échantillon, la présence des deux espèces de petits ruminants, la diversité des sites d'étude. En revanche, ce taux de 21,5 p. 100 a été largement supérieur à celui de 2,9 p. 100 obtenu au Cameroun chez des bovins qui avaient aussi avorté. Ceci s'explique par le fait que les réactions sont différentes selon les espèces.

L'étude a montré que la distribution des animaux séropositifs pour la fièvre $Q$ a varié en fonction des sites $(\mathrm{p}<0,001)$. Les taux ont été plus importants dans les sites de Nioro et de Kéniébougouwéré, ce qui pouvait s'expliquer par la présence de populations de petits ruminants plus importantes et par la conduite d'élevage de ces espèces dans ces sites (fréquence de la conduite groupée de troupeaux appartenant à plusieurs éleveurs du même village). Ces facteurs favorisent la transmission rapide de l'infection.

Les résultats ont montré un lien statistiquement significatif entre la fièvre $Q$ et le rang de portée $(p<0,001)$. Ce phénomène pourrait s'expliquer par le fait qu'une fois que l'animal rentre en contact avec le germe, il reste porteur pour le restant de sa vie.

Toutefois, les contagions au sein des troupeaux n'ayant pu être évaluées, il est probable que les intervalles de confiance relatifs aux autres facteurs aient été sous-estimés. Ceci incite à interpréter les résultats avec prudence et à approfondir l'analyse des effets du site et du rang de portée par la prise en compte des dépendances intra-troupeau.

L'évaluation de l'impact économique a permis d'estimer les pertes attribuées aux avortements. Elle a révélé un manque à gagner considérable pour les éleveurs, surtout dans un pays où les revenus sont assez faibles. Un meilleur contrôle de ces pertes permettrait, d'une part, d'augmenter les revenus des éleveurs et, d'autre part, de réduire le déficit en protéines animales (viande et lait).

\section{CONCLUSION}

Cette étude a rendu compte de la prévalence sérologique de la fièvre Q chez les petits ruminants de quatre régions du Mali. Les sites et le rang de portée ont été les seuls facteurs pour lesquels il a été possible de mettre en évidence une association statistique significative avec la maladie. L'étude a aussi permis d'estimer les pertes économiques directes enregistrées, résultant des avortements observés chez les petits ruminants infectés par la fièvre Q. Des recherches complémentaires intégrant le diagnostic moléculaire et des prélèvements sur des animaux témoins permettraient de confirmer l'implication de la fièvre $\mathrm{Q}$ dans les cas de pertes en reproduction chez les petits ruminants au Mali. En outre, des prélèvements réalisés à intervalles de deux à trois semaines permettraient d'évaluer la cinétique des anticorps des animaux chez lesquels ces pertes en reproduction ont été observées. L'étude a enfin permis d'établir que les pertes financières enregistrées suite aux avortements étaient considérables.

La fièvre $\mathrm{Q}$ constitue un problème de santé publique au Mali. Afin de mieux la contrôler chez les animaux et chez les humains, les services vétérinaires et de santé publique doivent renforcer leur collaboration qui passe nécessairement par la mise en place d'un programme national de lutte contre les zoonoses majeures en général et contre la fièvre $\mathrm{Q}$ en particulier.

\section{Remerciements}

Les auteurs adressent leurs sincères remerciements au Programme d'appui aux services agricoles et organisations paysannes (Pasaop) pour le financement de cette étude, aux structures techniques d'encadrement et aux éleveurs de petits ruminants pour la franche collaboration.

\section{BIBLIOGRAPHIE}

1. AKAKPO A.J., TEOU K.L., KPONMASSI T., ZELLER H.G., 1994. Epidémiologie des affections abortives des ruminants au Togo. Enquête sérologique sur la brucellose, la chlamydiose, la fièvre Q et la fièvre de la vallée du Rift. Biotechnologies du diagnostic et de la prévention des maladies animales. Paris, France, Aupelf-Uref, p. 125-137.

2. AUBERT M., ROUSSET E., 2003. Fièvre Q et élevage : sommes-nous démunis en matière vétérinaire ? In : Journée technique ovine NOE, Montpellier, France, 16 avr. 2003.

3. BARADJI I., BROUQUI P., 1999. Observations préliminaires sur la séroprévalence de la fièvre $Q$ chez les animaux domestiques au Mali. In : EUWOG-ASR joint meeting, Marseille, France, 14-16 juin 1999, $89 \mathrm{p}$.

4. BONFOH B., FANE A., STEINMANN P., HETZEL M., TRAORE A.N., TRAORE M., SIMBE C.F., IDRISS O.A., NICOLET J., AKAKPO J.A., FARAH Z., ZINSSTAG J., 2003. Qualité microbiologique du lait et des produits laitiers vendus au Mali et leurs implications en santé publique. Etud. Rech. Sahél. (8-9) : 19-27.

5. BOUVERY N.A., SOURIAU A., LECHOPIER P., RODOLAKIS A., 2003. Experimental Coxiella burnetii infection in pregnant goats: excretion routes. Vet. Res., 34: 423-433.

6. BUHARIWALLA F., CANN B., MARRIE T.J., 1996. A dog-related outbreak of Q fever. Clin. Infect. Dis., 23: 753-755.

7. CHARTIER C., CHARTIER F., 1988. Enquête séro-épidémiologique sur les avortements infectieux des petits ruminants en Mauritanie. Rev. Elev. Méd. Vét. Pays Trop., $41: 23-34$.

8. DAVID H.Z., PAMELA L., PEACOCK M.G., 1990. Ovine abortion caused by Coxiella burnetii. In: Laboratory diagnosis of livestock abortion, 3rd Ed. Ames, IA, USA, lowa University Press, 175 p.

9. EUZEBY J.P., 2001. Dictionnaire de bactériologie vétérinaire. http:// www.bacterio.cict.fr/bacdico/cvfrançais.html (juin 2009).

10. FONTAINE M., GIAUFFRET A., RUSSO P., DURAND M., 1975. Importance des troupeaux ovins dans l'épidémiologie de la fièvre Q. Méd. Mal. Infect., 5 : 445-449. 
11. FOURNIER P.E., MARRIE T.J., RAOULT D., 1998. Diagnosis of Q fever. J. Clin. Microbiol., 36: 1823-1834.

12. GARDON J., HERAUD I.M., LAVENTURE S., LADAM A., CAPOT P., FOUQUET E., 2001. Suburban transmission of Q fever in French Guiana: evidence of a wild reservoir. J. Infect. Dis., 184: 278-284.

13. GREENSLADE E., BEASLEY R., JENNINGS L.,WOODWARD A., WEINSTEIN P., 2003. Has Coxiella burnetii (Q fever) been introduced into New Zealand? Emerg. Infect. Dis., 9.

14. Guatteo R., BeAudeau F., JOly A., SEeGers H., 2006. L'infection des bovins par Coxiella burnetii. Point Vét., 37 : 62-66.

15. JOUBERT L., FONTAINE M., BARTOLI M., GARRIGUE G., 1976. La fièvre Q ovine, zoonose d'actualité de type professionnel, rural et militaire. Rev. Méd. Vét., $127: 361-381$.

16. JOURDAIN E., 2003. Etude des maladies abortives non réglementées chez les ongulés sauvages et domestiques de la réserve nationale de chasse et de faune sauvage des Bauges. Thèse Doct., Université ClaudeBernard, Lyon I, France, $161 \mathrm{p}$.

17. LANG G.H., 1990. Coxiellosis in animals. In: Marie T.J. Ed., The diseases: Q fever. Boca Raton, FL, USA, CRC Press, p. 23-48.

18. MANTOVANI A., BENAZZI P., 1953. The isolation of Coxiella burnetii from Rhipicephalus sanguineus on naturally infected pigs. J. Am. Vet. Med. Assoc., 122: 117-118.

19. MARRIE T.J., DURANT A., WILLIAMS T.C., MINTZ E., WAAG D.M., 1988. Exposure to parturient cats: A risk factor for acquisition of $Q$ fever in Maritime Canada. J. Infect. Dis., 158: 101-108.

20. MARRIE T.J., WILLIAMS J.C., SCHLECH W.F., YATES L., 1986. Q fever pneumonia associated with exposure to wild rabbits. Lancet, 22: 425427.

21. MATTHEWS J., 1999. Abortion. In: Matthews J., Ed., Diseases of goats, 2nd Edn. Paris, France, Blackwell Science, p. 22-36.

22. MAURIN M., RAOULT D., 1999. Q fever. Clin. Microbiol. Rev., 12: 518-553.

23. PEREZ-EID C., GILOT B., 1998. Les tiques : cycles, habitats, rôle pathogène, lutte. Méd. Mal. Infect., $28: 335-343$.

24. PINSKY R.L., FISHBEIN D.B., GREENE C.R., GENSHEIMER K.F., 1991. An outbreak of cat-associated Q fever in the United States. J. Infect. Dis., 164: 202-204.

25. PONCELET J.L., 1994. Les ricckettsioses transmises par les tiques. Bull. Group. Tech. Vét., 3 : 105-109.

26. QUIGNARD H., GERAL M.F., PELLERIN J.L., MILON A., LAUTIER R., 1982. La fièvre Q chez les petits ruminants. Enquête épidémiologique dans la région de Midi-Pyrenées. Rev. Méd. Vét., 133 : 413-422.
27. RAOULT D., MARRIE T., 1995. Q fever. Clin. Infect. Dis., 20: 489496.

28. REKIKI A., THABTI F., DLISSI I., 2005. Enquête sérologique sur les principales causes d'avortements infectieux chez les petits ruminants en Tunisie. Rev. Méd. Vét., 156 : 395-401.

29. RODOLAKIS A., BOUVERY N.A., 2000. Diagnostic de la chlamydiose et de la fièvre Q. Vet. Reprod., 2 : 29-33.

30. RODOLAKIS A., 1994. Chlamydiose et fièvre Q : agents d'avortements et zoonoses? Point Vét., 26 : 845-850,

31. ROUSSET E., RUSSO P., PEPIN M., RAOULT D., 2001. Epidémiologie de la fièvre Q animale. Situation en France. Méd. Mal. Infect., $31:$ 233-246.

32. ROUSSET E., DUQUESNE V., RUSSO P., THIERY R., 2007. La fièvre Q : problématique et risque sanitaire. Bull. Acad. Vét., 2 : 107.

33. ROUSSET E., RUSSO P., PEPIN M., RAOULT D., 2000. La fièvre Q : une zoonose encore mystérieuse. Bull. Group. Tech. Vét., 7 : 133-137.

34. RUSSO P., MALO N., THEVENOT C., 1981. La fièvre Q dans le département de Vienne. Cinétique des anticorps et avortement. Recl Méd. Vét., 157 : 585-589

35. SCHALCH L., RUSSO P., DE SA C., REYNAUD A., BOMMELI W., 1998. Combined tested of ruminant serum samples for Chlamydia psittaci and Coxiella burnetii specific antibody ELISA. In: 6th Congr. FeMe SPRum, Postojna, Slovenia, 14-16 May 1998, p. 541-518.

36. HANON J.B., UYTTENHOEF A., FECHER-BOURDEOIS $F$. KIERSCHVINK N., HAUBRUGE E., DUQUESNE B., SAEGERMAN C., 2009. Estimation des pertes économiques attribuées à la fièvre catarrhale ovine (sérotype 8) dans le sud de la Belgique durant la période 20062007. Rencontres Rech. Rumin. (16).

37. SCHELLING E., DIGUIMBAYE C., DAOUD S., NICOLET J., ZINSSTAG J., 2004. Séroprévalence des maladies zoonotiques chez les pasteurs nomades et leurs animaux dans le Chari-Baguirmi au Tchad. Med. Trop., 64 : 474-477.

38. STEIN A., RAOULT D., 1999. Pigeon pneumonia in Provence: A birdborne Q fever outbreak. Clin. Infect. Dis., 27: 617-620.

39. THIBON M., WILLERSS V., SOUQUE P., DOUTRY-VARSAT A., DUQUESNEL R., OJCIUS D.M., 1996. High incidence of Coxiella burnetii markers in a rural population in France. Eur. J. Epidemiol., 12: 509-513.

40. WORTHINGTON R.W., 2001. New Zealand is free from Q fever Surveillance, 28: 3-4.

Accepté le 12.09.2013 


\section{Summary}

Sidibe S.S., Coulibaly K.W., Dakouo M., Tarnagda Z., Sery A., Niang M., Traore K., Nantoume H., Diarra S., Seyni H. $\mathrm{Q}$ fever in small ruminants in Mali. Results of a serological survey

In Mali, the loss of reproduction is a major constraint to improving the productivity of small ruminants. The causes of these losses are still poorly known and include infertility, abortions, orchitis and stunting. The purpose of this study was to assess the seroprevalence of $\mathrm{Q}$ fever in small ruminant farms where cases of loss of reproduction had been observed, as well as financial losses linked to the recorded abortions. The survey was carried out during the period 2006-2009 in the agropastoral areas of Bougouni (Sikasso Region), Nioro (Kayes Region), Keniebougouwere (Segou Region) and Koro (Mopti Region). Out of 718 sera analyzed by indirect ELISA, $155(21.5 \pm 3 \%)$ tested positive for Coxiella burnetii. The prevalence rate varied according to the site and the birth rank. The highest prevalence rate was observed in Keniebougouwere $(35 \pm 6 \%)$, followed by Nioro $(28.5 \pm 7.5 \%)$ and Bougouni $(10.8 \pm 4.6 \%)$, and the lowest in Koro $(5.8 \pm 3.7 \%)$. This study revealed the presence of $\mathrm{Q}$ fever in small ruminants in Mali. Complementary investigations that include molecular diagnosis (PCR technique) might help understand the etiology of the disease involved in cases of loss of reproduction in small ruminants in Mali. The technical and economical analysis helped to assess the financial value of losses.

Keywords: Sheep - Goat - Q fever - Reproduction - Loss Serology - Mali.

\section{Resumen}

Sidibe S.S., Coulibaly K. W., Dakouo M., Tarnagda Z., Sery A., Niang M., Traore K., Nantoume H., Diarra S., Seyni H. Fiebre $\mathrm{Q}$ en pequeños rumiantes en Malí: resultados de una encuesta serológica

En Malí, las pérdidas relativas a la reproducción son los principales obstáculos para mejorar la productividad de los pequeños rumiantes. Entre las causas de estas pérdidas, aún poco conocidas, se encuentran, entre otros, infertilidad, abortos, orquitis y retraso del crecimiento. El propósito de este estudio fue evaluar la seroprevalencia de la fiebre $Q$ en fincas de pequeños rumiantes donde se observaron casos de pérdidas relacionadas con la reproducción, así como el valor económico de las pérdidas financieras relacionadas con los abortos registrados. El estudio se realizó durante el período de 2006 a 2009 en las zonas agropecuarias de las localidades Bougouni (región de Sikasso), Nioro (región de Kayes), de Keniebougouwere (región de Segou) y Koro (región de Mopti). De los 718 sueros analizados por el método de ELISA indirecto, $155(21,5 \pm 3 \%)$ fueron positivos para anticuerpos contra Coxiella burnetii. Esta prevalencia varió dependiendo del sitio y el rango de la camada. La mayor prevalencia se observó en Kéniébougouwèrè $(35 \pm 6 \%)$, seguido de Nioro $(28,5 \pm 7,5 \%)$, a continuación Bougouni $(10,8 \pm 4,6 \%)$ y la más baja en Koro $(5,8 \pm 3,7 \%)$. Estudios complementarios que incorporasen diagnósticos moleculares (técnica de PCR) podrían ayudar a dilucidar el papel etiológico de esta enfermedad en caso de pérdidas relacionadas a la reproducción actualmente registradas en los pequeños rumiantes en Malí. El análisis técnico-económico permitió evaluar el valor económico de las pérdidas.

Palabras clave: Ovino - Caprino - Fiebre Q - Reproducción Pérdida - Serología - Malí. 